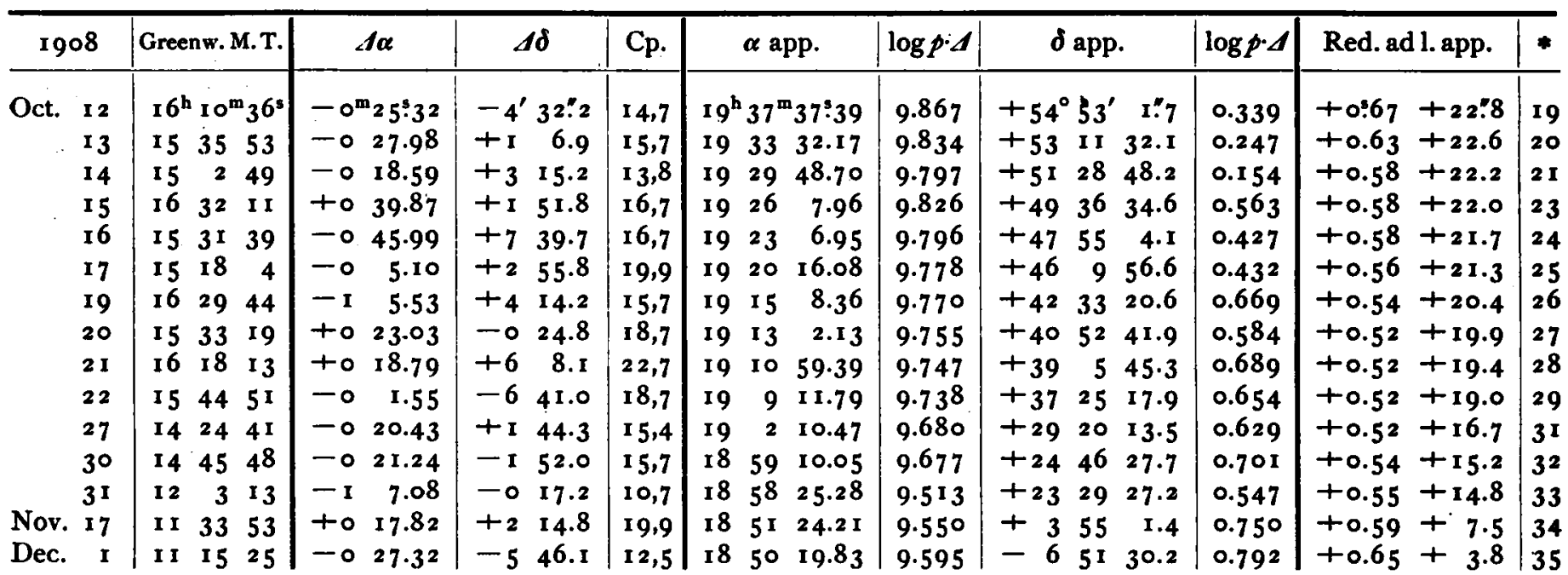

Right ascensions marked "were measured with the micrometer; all others by transits.

Mean places of comparison stars for the beginning of the year.

\begin{tabular}{|c|c|c|c|c|c|c|c|c|c|c|c|c|c|c|c|}
\hline$*$ & \multicolumn{3}{|c|}{$\alpha$} & \multicolumn{3}{|c|}{$\boldsymbol{\delta}$} & Authority & $*$ & \multicolumn{3}{|c|}{$\boldsymbol{\alpha}$} & \multicolumn{3}{|c|}{$\delta$} & Authority \\
\hline $\mathbf{I}$ & 6 & $h_{4}-1010$ & $\mathrm{~m}_{2} 2^{\mathrm{s}} .49$ & $-\mathbf{I} \mathbf{r}^{\circ}$ & & $49 ! 3$ & $B D-I 1^{\circ}{ }^{6} 59$, cp. with 2 & 19 & 19 & 38 & 2.04 & $+54^{\circ}$ & $57^{\prime}$ & I I". I & AG Cbr M. 6r 42 \\
\hline 2 & 6 & 44 & I 2.59 & - I I & 44 & 53.6 & AG Cbr M. & 20 & 19 & 33 & 59.52 & +53 & 10 & 2.6 & . 6.105 \\
\hline 3 & 6 & $4 I$ & 46.75 & - Io & I I & 53.9 & BD $-10^{\circ} 1683$, cp. with 4 & $2 \mathbf{I}$ & 19 & 30 & $6.7 \mathrm{I}$ & +51 & 25 & I 0.8 & $\mathrm{BD}+5 \mathrm{I}^{\circ} 26 \mathrm{r}_{3}, \mathrm{cp}$. with 22 \\
\hline 4 & 6 & & 8.84 & -10 & 13 & 28.9 & AG Cbr M. & 22 & 19 & 27 & 23.25 & +51 & $3^{2}$ & 0.4 & $1 / 2$ (AG Cbr M. $6043+$ \\
\hline 5 & 6 & & 8.49 & -8 & 33 & 8.6 & BD $-8^{\circ} 15^{29}, \mathrm{cp}$. with 6 & & & & & & & & Newcomb F.C. I 260) \\
\hline 6 & 6 & 35 & 37.72 & -8 & 24 & 36. 1 & AG Ott 2085 & 23 & I 9 & 25 & $27.5 \mathrm{I}$ & +49 & 34 & 20.8 & AG Bo 13032 \\
\hline 7 & 6 & 14 & 51.84 & +4 & 40 & 43.4 & AG Alb 2 I I 9 & 24 & 19 & 23 & 52.36 & +47 & 47 & 2.7 & - $\quad 12988$ \\
\hline 8 & 6 & 12 & $45 \cdot 44$ & +6 & 28 & 26.3 & Micr. comp. with 9 & 25 & 19 & 20 & 20.62 & +46 & 6 & $39 \cdot 5$ & 12922 \\
\hline 9 & 6 & I 1 & 1.02 & +6 & 34 & 0.1 & AG Lpz II 2752 & 26 & 19 & 16 & $13 \cdot 35$ & +42 & 28 & 46.0 & 12852 \\
\hline IO & 21 & 35 & 20.51 & +73 & I 5 & 14.6 & Micr. comp. with I I & 27 & 19 & 12 & $3^{8} \cdot 5^{8}$ & +40 & $5^{2}$ & 46.8 & $>\quad 12774^{\circ}$ \\
\hline I I & 21 & 30 & $37 \cdot 3^{6}$ & +73 & I 5 & 17.1 & AG Do & 28 & 19 & Io & 40.08 & +38 & 59 & 17.8 & AG Lu 8237 \\
\hline 12 & 2 I & I9 & 18 & +72 & 2 I & & $\mathrm{BD}+72^{\circ} 983$ & 29 & 19 & 9 & 12.82 & +37 & $3 \mathbf{I}$ & 39.9 & $\mathrm{BD}+37^{\circ} 337^{2}$, cp. with 30 \\
\hline I 3 & 20 & $5^{6}$ & 49.49 & +70 & 4 & 52.9 & AG Chri 3266 & 30 & 19 & 10 & 25.90 & +37 & 37 & 16.4 & AG Lu 8232 \\
\hline 14 & 20 & 41 & 41.45 & +68 & 50 & I I.I & $\mathrm{BD}+68^{\circ}$ I I $43, \mathrm{cp}$. with I 5 & 31 & 19 & 2 & $30.3^{8}$ & +29 & 18 & 12.5 & AG Cbr E. 9580 \\
\hline I 5 & 20 & 47 & 32.02 & +68 & 47 & 48.3 & AG Chri $323^{8}$ & 32 & 18 & 59 & 30.75 & +24 & 48 & $4 \cdot 5$ & AG Berl B 68 I I \\
\hline I 6 & 20 & 20 & 58.81 & +66 & 2 & 40.8 & Micr. comp. with I 7 & 33 & 18 & 59 & 31.81 & +23 & 29 & 29.6 & $\times \quad 6810$ \\
\hline 17 & 20 & 21 & 6.72 & +65 & 54 & 50.2 & Chri 28, A. N. ${ }_{428}$ & 34 & 18 & $5^{1}$ & 5.80 & +3 & 52 & 39.1 & AG Alb 6422 \\
\hline 18 & 20 & I 4 & $24 \cdot 3^{6}$ & +64 & $3 \mathbf{I}$ & $35 \cdot 7$ & AG Hels I I 245 & 35 & 18 & 50 & 46.50 & -6 & 45 & 47.9 & AG Ott 6449 \\
\hline
\end{tabular}

The positions of the stars from AG Do and AG Cbr M. (southern zone) were furnished through the courtesy of the directors of the observatories.

\title{
Ausbrüche der Sonnentätigkeit, beobachtet und gemessen an Sonnenflecken.
}

\section{Von Wilhelm Krebs.}

In A. N. 4270 sind Ausbruchserscheinungen an der Sonnenoberfläche, durch welche Fleckenmassen schlierenförmig emporgetrieben und über andere Fleckenpartien gelagert wurden, von mir beschrieben. Die Überlagerung wurde damals zwei Schlieren zu teil. Es erscheint von Wert hier nachzutragen, daß sie nahezu parallel bei beiden Schlieren und im wesentlichen von Westen nach Osten erfolgte. Dieser Richtung der Überlagerung entsprach bei der größeren Winkelgeschwindigkeit der höheren Schichten ein Zurückbleiben der von unten aufgestiegenen Massen. Durch dieses Zurückbleiben wird der Schluß auf Ausbruch dieser Schlieren noch erhärtet.

Das Zurückbleiben nach Osten steht in einem sehr einfachen Verhältnis zur Höhenänderung; dieses Verhältnis ist schon zur Korrektion der von mir in Nr. 4267 der $A$. N. veröffentlichten Berechnung der Tiefen-Unterschiede von Sonnenflecken verwendet. Im folgenden ist es noch etwas genauer formuliert. 
Nennen wir $u$ den der Zwischenzeit der Beobachtungen entsprechenden Rotationswinkel der Sonne, $H$ die als momentan erfolgt angesehene Höhenänderung und $S$ den beobachteten Betrag des Zurückbleibens, so ist $S=H \operatorname{tg} u$, wobei $u$ aus der beobachteten Bewegung des Fleckens $z \mathfrak{u}$ bestimmen ist. Als lineare Einheit ist im folgenden I Megameter $=1000 \mathrm{~km}$ angenommen. Die so erzielte Genauigkeit genügt für diese ersten Versuche auf einfachem planimetrischem Wege sich Rechenschaft zu geben über Höhen- und Tiefen-Unterschiede auf der Sonne.

Als Zwischenzeit $t$ ist die Zeitstrecke angenommen bis zu der nächstvorhergehenden Aufnahme, von der aus gemessen wurde. Die so erhaltenen Beträge geben deshalb nur ein Minimum der Höhenänderung $H$ an, wenn jene Ausgangsphase von den ausgemessenen Gebilden noch nichts erkennen ließ. Das gilt für die I908 Aug. 7 beobachteten beiden Schlieren. Von ihnen war zuletzt $121 / 2$ Stunden vor dieser Aufnahme nichts zu sehen gewesen.

Die Messungen dèr relativen Bewegung ergaben

für I, nördliche Schliere 52 Megameter

für II, südliche Schliere 90 》

Die Reduktion dieser durch die Perspektive beeinträchtigten Werte auf senkrechte Lage zur Gesichtslinie ergab $S=65$ Meg., bezw. $S=$ I 2 Meg. und damit für $u=7^{\circ} 5^{\prime}$ folgende Werte der Höhenänderung:

$$
\text { I } 506 \mathrm{Meg} . \quad \text { II } 872 \mathrm{Meg} \text {. }
$$

Diese Zahlen erscheinen reichlich groß, besonders da sie, wie erwähnt, als Mindestwerte aufzufassen sind. Aber sie erheben sich nicht über die Größenordnung der größten bisher gemessenen Protuberanzen-Ausbrüche am Sonnenrande, an denen ja auch nur Mindestwerte der auf die optische Ebene projizierten, über den Sonnenrand hinausragenden Strecken zur Messung gelangt sind.

rgo9 Mai r $3^{-14}$ gelang es, an einer ebenfalls schon auf die Westseite vom Mittelmeridian gelangten Sonnenfleckengruppe Schlieren zu verfolgen, deren eine, die westlichste, am 14 . sich ebenfalls über den Hauptteil der Gruppe legte. Die Verlagerung der Schlieren nach Osten wurde gemessen $={ }_{17.5}$ Meg., red. wegen Neigung zur Gesichtslinie $=22 \mathrm{Meg}$.

Die Berechnung ergab mit $u=I 5^{\circ} \mathrm{I} 4^{\prime}$ als Höhenänderung $82 \mathrm{Meg}$.

Dieselbe Stelle der Sonnenoberfläche gelangte am 4. Juni wieder auf den Mittelmeridian der der Erde zu- gekehrten Sonnenseite, ohne daß sie zunächst ein Fleckensignal erkennen ließ. Zuerst gelangte 1909 Juni $47^{\mathrm{h}} 35^{\mathrm{m}}$ M. E. Z. in Großflottbek eine neugebildete Gruppe zur Aufnahme. Ihre größte, von Westen nach Osten gehende Ausdehnung konnte auf mehr als $60 \mathrm{Meg}$. geschätzt werden. Sie war aber in starker Zunahme begriffen. Während der große Kopfleck im Westen nur unbedeutende Veränderungen zeigte, waren die übrigen, an seiner östlichen Rückseite sich anschließenden Teile der Gruppe von Tag zu Tag kaum wieder zu erkennen. Bei diesen bedeutenden Umgestaltungen ergaben sich Formen, die sichtlich auf Schlieren- und Wirbelbildung hinauskamen. Die wesentlichste Änderung ereignete sich im Iaufe von 1909 Juni 7 , als die Gruppe ungefähr auf den Meridian $45^{\circ}$ westlich vom Mittelmeridian angelangt war. Juni 6 um $18^{\text {h }} 30^{\mathrm{m}}$ M. E. Z. ließ jener Schweifteil ungefähr in seiner Mitte eine Knickung erkennen. Östlich dieser Mitte war die bis dahin östliche Richtung in eine südöstliche umgeändert. Juni $70^{\mathrm{h}} 20^{\mathrm{m}}$ M. E. Z. lagen die Hauptflecken dieses Schweifes in nahe der gleichen heliographischen Länge mit der Knickung. Um $6^{\mathrm{h}}$ abends waren sie ihr auf $1 /$ : des Weges bis zum Kopffleck nach westlicher Richtung vorangeeilt.

Aus diesen Wahrnehmungen ging hervor, daß der östliche Endteil der Sonnenfleckengruppe einem erheblich höheren Niveau angehörte als ihr westlicher Teil und besonders der Kopffleck. Damit stand in Übereinstimmung das vorherige rasche scheinbare Wachstum der Gruppe nach Osten hin. Es war offenbar veranlaßt durch ein Zurückbleiben der in lebhafter Umformung begriffenen Massen infolge raschen Aufsteigens $z u$ größeren Höhen, also durch eine echte Ausbruchserscheinung.

Zur Berechnung dieser Höhenänderung erwiesen sich nur zwei Morgenaufnahmen von 1909 Juni 5 und 6 als hinreichend genau. Es ergaben sich aus ihnen als Verlängerung des Endteils, infolge Zurückbleibens der östlichen Fleckenmassen, 40 Megameter. Daraus folgt $S=50$ Meg. und mit $u=14^{\circ} \circ^{\prime}$ als Höhenänderung (Ausbruchshöhe) $202 \mathrm{Meg}$.

Diese Angaben können bei der Ungenauigkeit der Messungen an freihändig projizierten oder nach dem Augenmaß entworfenen Fleckenzeichnungen keinen Anspruch auf quantitative Schärfe machen. Sie beanspruchen aber qualitative Geltung, als weitere Belege für die Möglichkeit, Ausbruchserscheinungen auf der Sonnenoberfläche auch teleskopisch nachzuweisen.

Großflottbek (Holstein), I 909 Juni I 7.

W. Krebs.

\title{
Photographische Beobachtungen des Perrineschen Kometen 1909 b.
}

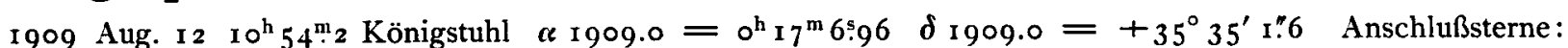 \\ AG Lu ro4, 2 I, AG Lei 98 . Kopff. Wolf. \\ 1909 Aug. $14{ }^{1} 3^{\mathrm{h}} 6^{\mathrm{m}} \cdot 7$ Greenwich $\alpha$ app. $=0^{\mathrm{h}} \mathrm{I}^{\mathrm{m}} 24^{\mathrm{s}} .06(9.297 \mathrm{n}) \quad \delta$ app. $=+36^{\circ} 35^{\prime} 14^{\prime \prime} 8$ (0.408) \\ Red. ad l. app. + $1997+3.4$. Cowell. \\ Inhalt zu Nr. 4349. A. Nekrassow. Über die Bahn des Kometen 181 I II. 65. - A. Hnatek. Eigenbewegungen von 68 Fixsternen. $69 .-$ \\ W. Wiindler. Beobachtungen von kleinen Planeten am Kreuzstabmikrometer. 75 . $-H$. $\mathrm{W}$. Bigeloul Observations of \\ Comets. 75. - W. Krebs. Ausbriche der Sonnentätigkeit, beobachtet und gemessen an Sonnenflecken. 77. - Photo- \\ graphische Beobachtungen des Perrineschen Kometen I909 b. 79.
}

Geschlossen 1909 Aug. I9. Herausgeber H. Kobold. Druck von C. Schaidt (G. \& A. Oheim). Expedition: Kiel, Moltkestr. 80. 